\title{
CFD-BASED MODELLING OF BUBBLE-PARTICLE COLLISION EFFICIENCY WITH MOBILE BUBBLE SURFACE IN A TURBULENT ENVIRONMENT
}

\author{
T. LIU, P. T. L. KOH and M. P. SCHWARZ
}

CSIRO Minerals, Clayton, Victoria 3169, AUSTRALIA

\begin{abstract}
Flotation modelling to date appears to have concentrated either on macroscale processes or on ideal microscale processes - there has been no attempt to integrate detailed models at different scales. In this paper, bubble-particle collision efficiency with mobile bubble surfaces in a turbulent flow is investigated from a multiscale modelling viewpoint and a general methodology for modelling of turbulent bubble-particle collision efficiency with mobile surfaces is presented. An integrated CFD-based scheme is developed. The method can also be applied to nonNewtonian slurries. Example simulations and comparisons are carried out to illustrate the method. Turbulence effects on particle-bubble collision efficiency are systematically studied using a 3D $k-\varepsilon$ turbulence model.
\end{abstract}

\section{NOMENCLATURE}

$\begin{array}{ll}E & \text { collision efficiency } \\ f_{i} & \text { hydrodynamic resistance function } \\ F & \text { total force } \\ F_{A} & \text { added mass force } \\ F_{B} & \text { buoyancy force } \\ F_{D} \text { mod } & \text { modified drag force } \\ F_{P} & \text { pressure gradient force } \\ k & \text { turbulence kinetic energy } \\ m_{p} & \text { particle mass } \\ N_{i}^{t} & \text { total number of particles } \\ N_{i} & \text { number of particles hitting the bubble } \\ r & \text { radial component } \\ R_{b} & \text { bubble radius } \\ R_{i}^{L} & \text { outer radius of an annulus } \\ R_{i}^{S} & \text { inner radius of an annulus } \\ R_{p} & \text { particle radius } \\ \mathrm{Re}_{\mathrm{Re}} & \text { bubble Reynolds number } \\ \mathrm{Re}_{t} & \text { turbulent Reynolds number } \\ p & \text { pressure } \\ u_{i} & \text { fluid velocity } \\ v_{i} & \text { particle velocity } \\ x_{i} & \text { particle position } \\ \varepsilon & \text { turbulence dissipation rate } \\ \lambda & \text { Kolmogorov length scale } \\ \mu & \text { dynamic viscosity } \\ v & \text { kinematic viscosity } \\ \theta & \text { tangential component } \\ & \end{array}$

\author{
$\rho \quad$ density \\ $\tau_{i j} \quad$ Reynolds stress
}

\section{INTRODUCTION}

Froth flotation is a complex three phase physico-chemical process which is used in mineral processing industries to selectively separate valuable minerals from gangue. The main functions of flotation machines are to keep particles in suspension, to disperse a sufficient amount of fine air bubbles to the pulp, and to maintain sufficient agitation for bubble-particle attachment to take place without detachment occurring before particles are collected in the froth. Development of flotation machines and optimisation of flotation processes have mainly been guided by experimental data and plant experience because modelling techniques have previously been unable to deal with the phenomenological complexity.

\section{Bubble-particle collision in flotation}

The motions of air bubbles and solid particles and their interactions in a flotation cell are the most important phenomena governing a flotation process. Flotation rate is related to cell design; to particle hydrophobicity, size and density; to gas flow rate; to bubble size, velocity and mobility; and to turbulence intensity and structure. Experiments reveal that increase in particle hydrophobicity, gas flow rate, bubble mobility and electrolyte concentration result in an increase in the flotation rate of all particles. Increase in agitation results in improvement in the flotation rate of fine and intermediate particles but decreases the flotation rate of coarse particles. These experiments indicate that considerations of agitation/turbulence and bubble mobility are required for accurate prediction of flotation rate (Pyke et al., 2003). Research related to various microscale processes occurring in flotation has been considered only under some ideal assumptions (Sherrell, 2004). It is important to note that there is virtually a complete lack of experimental data on collection processes in stirred turbulent conditions in which all variables are well controlled, i.e. particle and bubble sizes, gas flow rate, bubble velocity and mobility.

In recent years, researchers have started to use computational fluid dynamics (CFD) modelling of mechanically stirred flotation cells to study the complexity of three-phase flows within flotation cells (Koh and Schwarz, 2000, 2003, 2005). In CFD modelling, a flotation cell is discretized into individual finite volumes where local values of flow fields are calculated. The detailed understanding of flow gained using this approach allows modification to existing equipment and operations to improve flotation performance. Also this information 
provides a possibility to investigate bubble-particle interactions in a real environment. CFD modelling has become a popular method due to its informative results and low labour and equipment costs, as a result of fast development in turbulence modelling and in computer speed and capacity.

Surface forces are relatively short range and have little influence on the bubble-particle collision efficiency (hydrodynamic forces dominate this process), but have a strong influence on attachment and stability efficiencies, e.g. at high particle hydrophobicity and high electrolyte concentration, attachment and stability efficiencies reach their maximum values. Once chemistry has been optimised, further improvement to flotation can only be achieved by the enhancement of bubble-particle collision frequency and efficiency. Bubble-particle collision efficiency is a correction factor that accounts for the tendency of particles to follow streamlines around the bubble and hence avoid collisions. This paper is a fundamental study of bubble-particle collision efficiency in turbulent environments with mobile bubble surface. This investigation will describe the steady RANS and Lagrangian particle methods used, and will demonstrate the validity of computer model by comparing numerical results with experimental data found from literature. The proposed model is more comprehensive than any existing description as it takes into account the effects of bubble mobility, bubble surface effect on particle drag forces and turbulence on collision processes.

\section{Effect of bubble surface mobility on collisions}

In the investigations of bubble-particle collisions, most efforts have been focused on the regime in which the bubble surface is assumed to be immobile because of adsorption of surfactants intentionally added or of impurities. Schulze (1992) mentioned that the degree of bubble surface retardation is not solved and uncertainties exist when collision theory is applied in an industrial situation. Only a few papers were published in theoretical and experimental investigations of bubble-particle collisions under the conditions of mobile bubble surfaces (Hewitt et al., 1994; Dai et al., 1998; Nguyen, 1998). Mobile bubble surfaces increase critical thickness for rupture of the film between particle and bubble and enhance bubble-particle collisions (Schulze, 1992). An experimental study by Sam et al. (1996) showed that the surface of sufficiently large bubbles cannot be completely retarded and the bubble surface can be partially mobile. During bubble rise in a surfactant solution, the surface contaminants are swept to the rear surface of the bubble. The forward part of bubble surfaces can remain mobile even when the bubbles reach terminal velocities. This paper focuses on the collision enhancement due to bubble surface mobility and turbulence.

\section{Inertial forces in collisions}

Inertial forces are usually neglected because the traditional assumption of surface retardation for small to intermediate-size bubbles in the presence of surfactants. In a turbulent flotation cell, the particle and bubble velocities are high and inertial forces may not be negligible (Dobby \& Finch, 1986; Luttrell \& Yoon, 1992). The essential role of inertial particle deposition has been verified and described quantitatively for cases of Stokes number above the critical value (Dobby \& Finch, 1987). Dai et al. (1998) demonstrated that inertial forces are not negligible even at subcritical Stokes number under the condition in which the bubble surface is mobile. While the bubble-particle collision efficiency increases with the Stokes number during the particles approach to the bubble as a result of inertial deposition, as the particle streamlines around the bubble, inertial forces are a negative effect on the particlebubble collisions if the bubble surface is mobile. But the prediction of Dai et al. (1998) is valid only for Stokes numbers less than 0.27 .

\section{Bubble wall effects on collisions}

Bubble surface disturbs creeping flows when a particle approaches the bubble surface. This particle-wall interaction (the bubble wall effect) is known as the shortrange hydrodynamic interaction and is a function of particle-wall distance (Happel and Brenner, 1963). The Stokes drag force has been modified by Nguyen and Evans (2002):

- In the radial direction (the line joining the bubbleparticle centres): $\quad F_{r}=-6 \pi \mu R_{p} v_{r} f_{1}+6 \pi \mu R_{p} u_{r} f_{2}$

- In the tangential direction:

$$
F_{\theta}=-6 \pi \mu R_{p} v_{\theta} f_{3}+6 \pi \mu R_{p} u_{\theta} f_{4}
$$

where $R_{p}$ is the particle radius; $\mu$ is the fluid dynamic viscosity; $v$ and $u$ are the particle and fluid velocities, respectively; the subscript $r$ and $\theta$ describe the radial and tangential components of the drag forces and the particle and fluid velocities, respectively; the functions $f_{i}$ ( $i=1-4)$ describe the hydrodynamic resistance functions. For the interactions between a solid particle and non-slip or slip surface in Newtonian fluids, all four hydrodynamic resistance functions are available as a function of the separation distance between the particle and the surface (Goldman et al., 1967a, b; Goren and O’Neill, 1971; Happel and Brenner, 1963; Nguyen and Evans, 2002; 2004; Nguyen and Jameson, 2005). Dai et al. (1998) studied the bubble wall effect on drag forces only at touch points of particles and bubbles. Nguyen (1998) considered the resistance function as a constant even though it is highly dependent on the separation distance between the particle and surface. The resistance increases rapidly and dramatically as a particle approaches a bubble. In this paper, a dynamic resistance function is employed to accurately describe the bubble wall effect on drag force.

\section{Turbulence effect on collisions}

In a turbulent flow, energy cascades from the largest eddies to the smallest where it is dissipated as heat. The smallest eddies are characterised by the Kolmogorov length scale:

$$
\lambda=\left(\frac{v^{3}}{\varepsilon}\right)^{1 / 4}
$$

where $v$ is kinematic viscosity of the fluid and $\varepsilon$ is dissipation rate of turbulence energy.

Turbulence energy contained in eddies larger than the bubble diameter mainly contribute to bubble displacement (Hop et al., 1996). Turbulence energy contained in smaller eddies contributes to turbulent agitation in the boundary layer of a bubble (Gammelsater et al., 1997) and to fluctuations in particle motion. Even for $1 \mathrm{~mm}$ diameter bubbles, the local flow around a bubble appears to be turbulent and all transport processes are affected. Flotation rate is expected to be increased due to turbulence effects. 
Although Johansen (1995) and Gammelsater et al. (1997) studied bubble-particle collisions in a turbulent flow using 2D CFD simulations, they did not consider the bubble surface effect on particle drag coefficients. Moreover, the bubble surface effect on particle drag coefficients cannot be accurately predicted using a 2D numerical simulation (Cheung et al., 2001).

Modelling turbulent particle motion is very complex. Different assumptions are made with respect to the features of turbulence in order to simplify the problem. In this paper, turbulent flow is assumed as 3D isotropic homogeneous and stationary turbulence. Turbulence is a 3D manifestation of inertia and frictional forces caused by the increases in vorticity and strain, which are due to the partition of mechanical energy into rotational and translational energy. The influence of turbulence on small immersed particles is a convective phenomenon that leads to random motion of particles through the flow field. In modelling turbulent particle motion, it is essential to understand the interaction between eddies and particle. A stochastic model is used to produce a fluctuating flow field based on steady RANS modelling of a turbulent flow; then the flow is used to calculate particle trajectories using a Lagrangian approach.

\section{CFD-BASED MODELLING STRATEGY AND FORMULATION}

Macroscale flow fields in a turbulent flotation cell are influenced by flotation cell size and structure, inlet and exit configurations, impeller shape and tip speed, and air nozzle structure and air flow rate. In a typical industrial scale flotation cell, there are millions of bubbles and particles. Therefore, it is impossible to use a direct 3D numerical modelling approach resolving eddy, bubble and particle scales. A more practical approach is to develop a CFD model using time-averaged RANS and multi-fluid techniques through suitable microscale closure relations. Koh and Schwarz (2000, 2003) investigated the relationships between operating and design variables and macroscale flow fields using CFD modelling. Figure 1 illustrates the predicted flow field in a Denver type flotation cell. This technique combined with a population balance model can be implemented to calculate flow fields and bubble size distribution in a turbulent flotation cell. Briefly, the multiphase flow equations for the conservation of mass, momentum and turbulence quantities have been solved using the Eulerian-Eularian approach. Three-dimensional distributions of turbulence dissipation rate, turbulence kinetic energy, and air volume fraction can also be obtained from the CFD model. Figure 2 shows the distribution of turbulence dissipation rates corresponding to different impeller speeds.

The flow fields calculated from CFD models (Koh and Schwarz, 2000; 2003) are different from the assumptions of conventional predictive formula for bubble-particle collision efficiency (Nguyen and Kmet, 1992; Dai et al., 1998). In conventional predictive formulas, bubbles are assumed to rise in a quiescent liquid. Bubble rise velocity and Reynolds number are determined only by bubble size and fluid properties. The velocity distribution of the fluid near a bubble is described under the assumption that the flow is a potential flow by Dai et al. (1998). The particle trajectories are calculated considering only interceptional, inertial and partial wall effects. The bubble wall effect is considered only at the touch point of the bubble and particle.

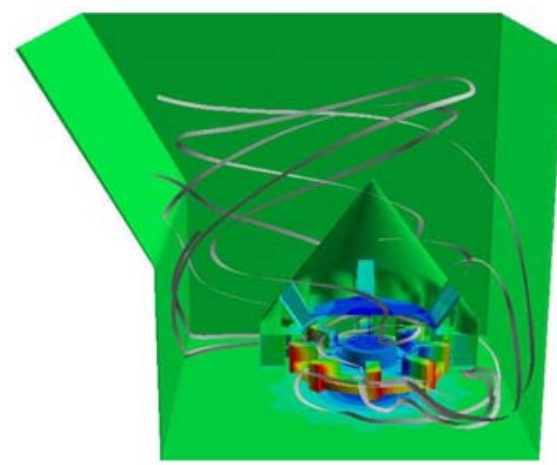

Figure 1: Predicted streamtraces in a Denver type flotation cell (Koh \& Schwarz, 2003)

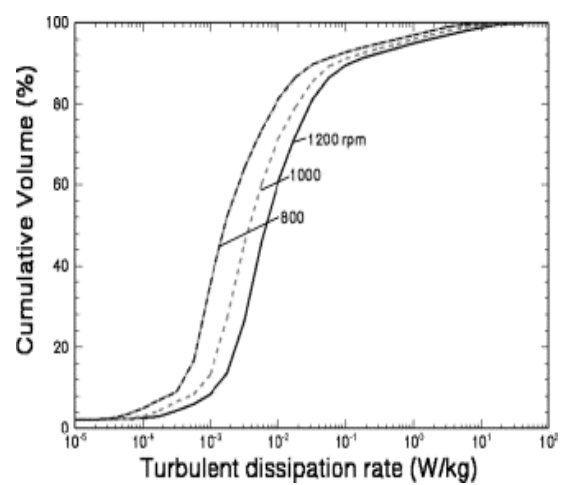

Figure 2: Predicted turbulence dissipation rate (Koh \& Schwarz, 2003)

In this paper, a numerical experiment is designed for the calculation of bubble-particle collision efficiency in a turbulent environment based on the local flow in a turbulent flotation cell. The computational domain used is shown in Figure 3.

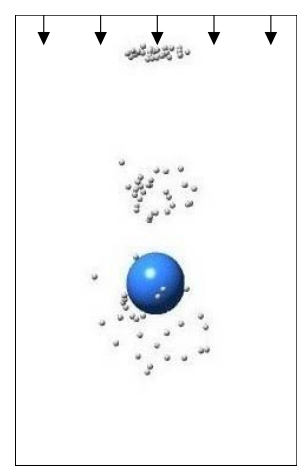

Figure 3: Particle-tracking scheme for analysing bubbleparticle collision efficiency

A three-dimensional model is set up using the commercial CFD package ANSYS CFX-5. The flow field is specified at the inlet, where velocity (corresponding to fluid velocity with respect to a rising bubble), turbulence intensity and length scale, or equivalently the turbulence kinetic energy $k$ and its dissipation rate $\varepsilon$, are specified based on the CFD model of a flotation cell. The turbulence field is calculated using a $k-\varepsilon$ model. The effect of turbulence on particles is incorporated using a 
randomly generated Gaussian turbulence field which is added to the mean flow field.

Because particles travel randomly in a turbulent flow, the bubble-particle collision efficiency must be defined in a slightly different way from the usual equation. A series of annuli with width equal to the bubble radius are defined at the inlet as shown in Figure 4. The collision efficiencies for particles originally released from each of these annuli are calculated. The total equivalent turbulent collision efficiency is defined as follows (Gammelsater et al., 1997):

$$
E=\sum_{i} E_{i}=\sum_{i} \frac{N_{i}}{N_{i}^{t}} \frac{\left(R_{i}^{L}\right)^{2}-\left(R_{i}^{S}\right)^{2}}{R_{b}^{2}}
$$

where $N_{i}^{t}$ is the total number of particles released from the annulus with radius of $R_{i}^{L}$ and $R_{i}^{S}$, and $N_{i}$ is the number of particles hitting the bubble.

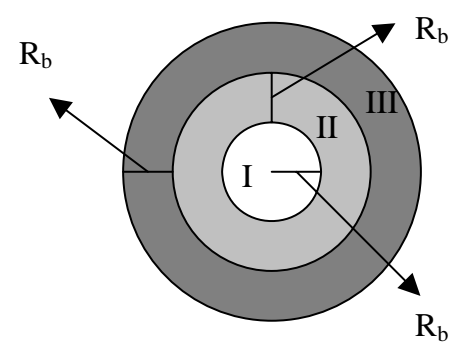

Figure 4: Particle release annuli at inlet

\section{Turbulent fluid model}

The grid for the computational domain in Figure 3 is a 3D body fitted mesh with several grid points inside the bubble's boundary layer: the grid is finest in the region near the surface of bubbles. The computational domain is assumed to be a cylinder with dimensions: length $\times$ diameter $=6 \times 8 \mathrm{~mm}$. The bubble diameter is $0.77 \mathrm{~mm}$. The diameter of the computational domain is large enough that the cylinder wall effect on bubble-particle collisions should be negligible. The flow domain was discretized into a non-uniform mesh of 3.84 million cells. 500 particles are used to calculate the bubble-particle collision efficiency. The initial velocity of particles is the same as the local fluid velocity. The bubble and domain walls are assumed as free slip. The flow is governed by RANS and mass continuity equations:

$$
\begin{aligned}
& \frac{\partial \bar{u}_{i}}{\partial t}+\frac{\partial\left(\bar{u}_{i} \bar{u}_{j}\right)}{\partial x_{j}}=-\frac{\partial \bar{p}}{\partial x_{i}}+\mu \frac{\partial^{2} \bar{u}_{i}}{\partial x_{i} \partial x_{j}}-\frac{\partial \tau_{i j}}{\partial x_{j}} \\
& \frac{\partial \bar{u}_{i}}{\partial x_{i}}=0
\end{aligned}
$$

The bar represents time-averaged velocity. The Reynolds stresses in Equation 1:

$$
\tau_{i j}=\overline{\left(u_{i}-\bar{u}_{i}\right)\left(u_{j}-\bar{u}_{j}\right)}
$$

are unknown and must be modelled. The present study uses the $k-\varepsilon$ model to describe the Reynolds stresses.

\section{Particle model}

As discussed in the previous section, a $k-\varepsilon$ model solves the turbulent flow. The flow field is then used to calculate particle trajectories and collision efficiencies. This study determines particle trajectories with a Lagrangian method. The interaction between the carrier fluid and particles has been treated using one-way coupling assuming that the effect of particles on the turbulent flow is negligible.

The Lagrangian method computes the trajectory of each particle by solving the momentum equations based on Newton's second law:

$$
\begin{aligned}
& \frac{\mathrm{d}\left(m_{p} v_{i}\right)}{\mathrm{d} t}=\sum F_{j} \\
& \frac{\mathrm{d} x_{i}}{\mathrm{~d} t}=v_{i}
\end{aligned}
$$

Momentum is transferred between fluid and particles through inter-phase drag and lift forces. The current study considers a modified drag force, which reflects the bubble surface effect on particle drag force, buoyancy force, added mass force and pressure force. So the term on the right side in Equation (4) can be expressed as:

$$
\sum F_{j}=F_{D}^{\bmod }+F_{B}+F_{P}+F_{A}
$$

where $F_{B}, F_{P}$ and $F_{A}$ are buoyancy force, pressure gradient force and added mass force, $F_{D}^{\text {mod }}$ is the modified drag force due to the effect of bubble surface. In turbulent tracking, the instantaneous fluid velocity is decomposed into mean and fluctuating components. The fluctuating component of the fluid velocity causes the dispersion of particles in the turbulent flow. A stochastic approach (Gosman and Ioannides, 1981) is adopted for the estimation of fluid fluctuating velocities.

Due to bubble surface effect on particle drag force and small particle Reynolds number, the modified drag force is expressed as follows:

$F_{D}^{\bmod }=F_{r}^{\bmod }+F_{\theta}^{\bmod }=-6 \pi \mu R_{p}\left(f_{1} v_{r}-f_{2} u_{r}+f_{3} v_{\theta}-f_{4} u_{\theta}\right)$

where $\mu$ is dynamic viscosity, $R_{p}$ is radius of particle, $v_{r}, u_{r}, v_{\theta}$ and $u_{\theta}$ are velocities of particles and fluid in normal and tangential directions, $f_{i}(i=1-4)$ are correction factors, which characterise the short range hydrodynamic interaction between particle and bubble surface. Correction factor functions mentioned in above section are employed. This study applies dynamic correction functions with respect to the distance between the bubble and particles. In fact, $F_{D}^{\bmod }$ goes to a finite value as particles approach to the bubble although $f_{1}$ goes to infinity (Mackay et al., 1963).

\section{RESULTS AND DISCUSSIONS}

The above numerical method has been used to study bubble-particle collisions in a turbulent flow. To demonstrate that a 3D CFD numerical simulation can accurately predict bubble-particle collision efficiency, a numerical experiment is designed to calculate bubbleparticle collision efficiency in a quiescent environment. The predictions are compared with experimental data (Dai et al., 1998). Then the method is applied to turbulent bubble-particle collisions.

\section{D numerical simulation for bubble-particle collision efficiency in a quiescent environment}

Bubble-particle collision efficiency is studied using numerical solutions of Navier-Stokes equations for the flow around a bubble. The flow is assumed steady and trajectories of individual particles are calculated to obtain actual bubble-particle collision efficiencies. The bubble surface is assumed to be spherical and free slip. The flow fields are obtained by a commercial CFD package ANSYS 
CFX-5. The fluid (water) velocity at the inlet is $0.196 \mathrm{~m} / \mathrm{s}$, which represents the bubble rising velocity. The particles used as samples are spherical quartz and galena particles with identical sizes. The densities of quartz and galena particles are $2650 \mathrm{~kg} / \mathrm{m}^{3}$ and $7500 \mathrm{~kg} / \mathrm{m}^{3}$, respectively. Figure 5 shows comparisons between the predicted bubble-particle collision efficiencies and those of current popular formula (mobile surface) and experimental data published by Dai et al. (1998). The computational results are in good agreement with the experimental data for particles with small Stokes number. This Lagrangian model can thus be used with confidence for investigating bubble-particle collision efficiency for particles with large Stokes number. Figure 6 shows the predicted results for galena particles with large Stokes number and quartz particles with small Stokes number. From the results, it can be seen that the bubble surface effect is more significant for low density particles. There is an interesting phenomenon for particles with small Stokes number: the higher the particle density, the lower the collision efficiency is. However, for particles with larger Stokes number, the higher the particle density, the higher the collision efficiency is. The phenomenon results from the action of inertia on the particles near a bubble with mobile surface, a centrifugal force, which opposes depositions. The proposed method is a general methodology. It can be applied to particles with large Stokes number, turbulent flow or non-Newtonian flow.

Collision Efficiency (Quartz, mobile surface bubble, Re =151)

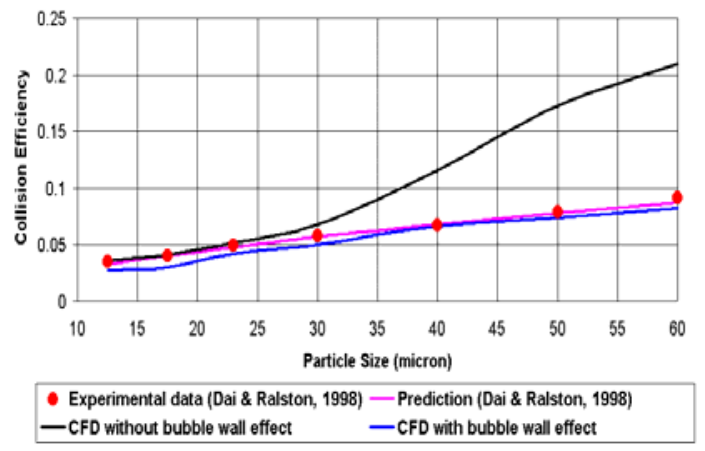

Figure 5: Comparison of collision efficiencies of Quartz particles

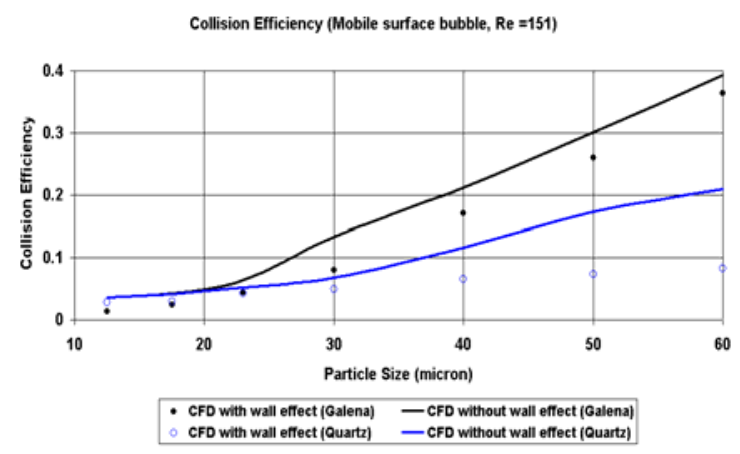

Figure 6: Comparison of collision efficiencies of Galena and Quartz particles
3D numerical simulation for bubble-particle collision efficiency in a local turbulent environment

In a real flotation cell, bubbles rise and interact with particles in a turbulent flow rather than in a quiescent fluid. A 3D model of bubble-particle collision in a turbulent flow is set up using the CFD package ANSYS CFX-5 based on the macroscale CFD simulation. The flow conditions are specified at the inlet, where velocity (corresponding to relative fluid velocity with respect to a rising bubble), the turbulence kinetic energy $k$ and its dissipation rate $\varepsilon$ are specified based on the CFD model of a flotation cell as the main turbulence is generated by the rotation of impellers. The turbulent field is calculated using the $k-\varepsilon$ model. The effect of turbulence on the particles is incorporated using a random generated Gaussian turbulent field which is added to the mean flow field. The turbulent Reynolds number is defined by:

$$
\operatorname{Re}_{t}=\frac{k^{2}}{\varepsilon v}
$$

where $v$ is kinematic viscosity.

The simulation results of collision efficiency with bubble Reynolds number 151 and different turbulent Reynolds numbers is shown in Figure 7. In the computational domain, main turbulence is the inflow turbulence generated by impellers. Therefore, the local turbulent Reynolds numbers can be high although the bubble Reynolds number is low. Particles flow from top to bottom. Snapshots at three time steps are shown in Figure 3. The diameters of bubble and quartz particles are 0.77 $\mathrm{mm}$ and $23 \mu \mathrm{m}$, respectively. Due to the turbulence, quartz particles do not follow streamlines. In order to obtain the accurate bubble-particle collision efficiency, 500 particle samples are used to calculate bubble-particle collision efficiency for different annuli at the inlet. Figure 7 shows the CFD predicted turbulent collision efficiency.

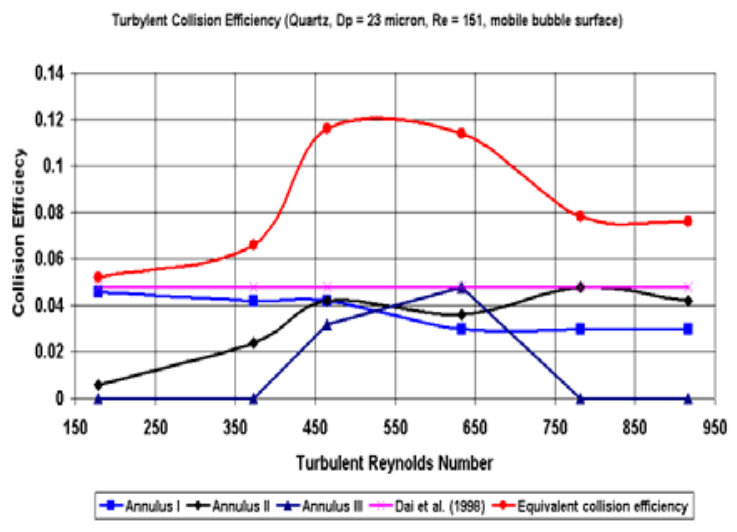

Figure 7: Comparisons between CFD predicted turbulent collision efficiencies and laminar collision efficiencies

The turbulence enhances the collection efficiency. As turbulence intensity increases, equivalent bubble-particle collision efficiency increases to a maximum and then decreases after a critical value. Considering that turbulence may increase the detachment rate, it is necessary to find an optimal turbulence intensity or impeller speed in real practice. At this stage, no experimental data regarding turbulent collision efficiencies have been published because of the difficulty of designing a well controlled experiment to measure 
these values. A direct numerical simulation is being developed to confirm the results.

\section{CONCLUSIONS}

This paper describes how to use a CFD numerical simulation with Lagrangian particle model to calculate bubble-particle collision efficiency in a turbulent environment. The bubble-particle collision efficiencies with large particle Stokes number, mobile bubble surface and in a 3D homogeneous isotropic turbulent flow are calculated firstly. By comparing the computed results with experimental data from the literature, the CFD simulation is shown to be a successful tool that correctly predicts the bubble-particle collision efficiency in a quiescent environment. The CFD method was then applied to the investigation of bubble-particle collision efficiency in a turbulent flow. The simulation results are qualitatively consistent with the real observations. Further direct numerical simulations of bubble-particle collision efficiency in a turbulent flow and bubble rising phenomenon in a turbulent flow are underway. This study indicates that micro-scale simulations of bubble and particle behaviour, coupled to macro-scale CFD simulations of flotation cells, provides a powerful tool predicting the flotation rate in a real flotation cell.

\section{REFERENCES}

CHEUNG, A. K. W., TAN. B. T., HOURIGAN, K. and THOMPSON, M. C., (2001), "Interference drag between spherical and cylindrical particles in Stokes flow", $14^{\text {th }}$ Australian Fluid Mechanics Conference, Adelaide, Australia, December 10-14.

DAI, Z., DUKHIN, S., FORNASIERO, D. and RALSTON, J., (1998), "The inertial hydrodynamic interaction of particles and rising bubbles with mobile surfaces”, J. Colloid Interface Sci., 197, 275-292.

DOBBY, G. S. and FINCH, J. A., (1986), “A model of particle sliding for flotation size bubbles”, J. Colloid Interface Sci., 109, 493-498.

DOBBY, G. S. and FINCH, J. A., (1987), "Particle-size dependence in flotation derived from a fundamental model of the capture process”, Int. J. Mine. Process., 21, 241260.

GAMMELSATER, R., BECH, K. and JOHANSEN, S. T., (1997), "Enhanced flotation of inclusions to bubbles due to turbulence", In: Huglen, R. (Eds.), Light Metals, TMS, 1007-1011.

GOREN, S. L. and O'NEILL, N. E., (1971), "Hydrodynamic resistance to a particle of a dilute suspension when in neighbourhood of a large obstacle", Chem. Eng. Sci., 26, 325-338.

GOLDMAN, A. J., COX, R. G. and BRENNER, H., (1967a), "Slow viscous motion of a sphere parallel to a plane wall - I Motion through a quiescent fluid”, Chem. Eng. Sci., 22, 637-651.

GOLDMAN, A. J., COX, R. G. and BRENNER, H., (1967b), "Slow viscous motion of a sphere parallel to a plane wall - II Couette flow”, Chem. Eng. Sci., 22, 653660.

GOSMAN, A. D. and IOANNIDES, E., (1981), "Aspects of computer simulation of liquid-fuelled combustors”, AIAA Paper No. 81-0323.

HAPPEL, J. and BRENNER, H., (1963), "Low Reynolds number hydrodynamics”, Martinus Nijhoff Publishers, Hague, Netherlands.
HEWITT, D., FORNASIERO, D. and RALSTON, J., (1994), "Bubble particle attachment efficiency", Miner. Eng., 7, 657-665.

HOP, B. I., JOHANSEN, S. T. and RASCH, B., (1996), "A 3D numerical model for removal of inclusions to gas bubbles”, Light Metals, The Minerals, Metals and Materials Society, 1027-1031.

JOHANSEN, S.T., FREDRIKSEN, A. and RASCH, B., (1995), "Particle flotation to bubbles in rotor-stirred reactors for melt treatment”, Light Metals, The Minerals, Metals and Materials Society, 1203-1206

KOH, P. T. L., MANICKAM, M. and SCHWARZ, M. P., (2000), "CFD simulation of bubble-particle collisions in mineral flotation cells”, Miner. Eng., 13, 1455-1463.

KOH, P. T. L. and SCHWARZ, M. P., (2003), "CFD modelling of bubble-particle collision rates and efficiencies in a flotation cell”, Miner. Eng., 16, 10551059.

KOH, P. T. L. and SCHWARZ, M. P., (2005), "CFD modelling of bubble-particle attachments in a flotation cell”, Centenary of Flotation Symposium 2005, Brisbane, Australia

LUTTRELL, G. H. and YOON, R. H., (1992), "A hydrodynamic model for bubble particle attachment”, $J$. Colloid Interface Sci., 154, 129-137.

MACKAY, G. D. M., SUZUKI, M. and MASON, S. G., (1963), "Approach of a solid sphere to a rigid plane interface, Part 2”, J Colloid Sci., 18, 103-104.

NGUYEN, A. V., (1998), "Particle-bubble encounter probability with mobile bubble surfaces”, Int. J. Miner. Process., 55, 73-86.

NGUYEN, A. V. and EVANS, G. M., (2002), "Axisymmetric approach of a solid sphere toward a nondeformable planar slip interface in the normal stagnation flow - development of global rational approximations for resistance coefficients”, Int. J. Multiphase Flow, 28, 13691380.

NGUYEN, A. V. and EVANS, G. M., (2004), "Exact and global rational approximate expressions for resistance coefficients for a colloidal solid sphere moving in a quiescent liquid parallel to a slip gas-liquid interface”, $J$. Colloid Interface Sci., 273, 262-270.

NGUYEN, A. V. and JAMESON, G. J., (2005), "Sliding of fine particles on the slip surface of rising gas bubbles: Resistance of liquid shear flows", Int. J. Multiphase Flow, 31, 492-513.

NGUYEN, A. V. and KMET, S., (1992), "Collision efficiency for fine mineral particles with single bubble in a countercurrent flow regime”, Int. J. Miner. Process., 35, 205-223.

PYKE, B., FORNASIERO, D. and RALSTON, J., (2003), "Bubble particle heterocoagulation under turbulent conditions”, J. Colloid Interface Sci., 265, 141-151.

SAM, A., GOMEZ, C. O. and FINCH, J. A., (1996), "Axial velocity profiles of single bubbles in water/froth solutions”, Int. J. Miner. Process., 47, 177-196.

SCHULZE, H. J., (1992), "Probability of particle attachment on gas bubbles by sliding”, Adv. Colloid Interface Sci., 40, 283-305.

SHERRELL, I. M., (2004), "Development of a flotation rate equation from first principles under turbulent flow conditions", PhD Thesis, Virginia Polytechnic Institute and State University, USA 